\title{
Bioartificial Organs: Ongoing Research and Future Trends
}

\author{
Loredana De Bartolo ${ }^{a}$ Diego Mantovani ${ }^{b}$ \\ anstitute on Membrane Technology, National Research Council of Italy, ITM-CNR, Rende, Italy; \\ b Laboratory for Biomaterials and Bioengineering, Laval University and CHU de Quebec Division of \\ Regenerative Medicine, Quebec, QC, Canada
}

\section{Keywords \\ Bioartificial organs · Biomaterials · Tissue engineering · \\ Biofabrication}

Healthcare surgery treatment for patients with tissue loss or end-stage organ failure is increasing due to the aging of the worldwide population and the progression of pathologies limiting or affecting organ functionality. Transplantation certainly represents the gold standard approach to saving life, but is limited by the scarce availability of organs and the unplanned availability of donors. As a direct consequence, there has been a growing demand from surgery for biological substitutes able to restore, maintain temporarily, or improve organ function. A device available on demand that can be implanted or integrated into a human body - interfacing with living tissue - offers the possibility to restore defective/failed organs, either temporarily or permanently. Recent advances in biomaterials, innovation in processing technologies, highly durable devices, progresses in decellularization of animal tissue and organs, and cell-based therapies have brought bioartificial organs closer to realization in clinics. Physiologically relevant organ substitutes result from the merging of heterogeneous cell types with multifunctional materials to recapitulate native organ geometries, components, and functions. On one hand, the main concern for bioartificial organs is the assembling process of heterogeneous living cells in predesigned architectures, including hierarchical vascular, neural, and/or lymphatic networks, thus guaranteeing the expected functionality. On the other hand, multiple bioengineering approaches include bioprinting organs and tissues, recellularization strategies, cellular repair or regeneration, and new functional biomaterials for the next generation of devices. Dynamic systems, such as bioreactors, have been developed and optimized to provide adequate oxygenation, and guarantee transport of nutrients to, and metabolic products from, the tissue. Altogether, these systems offer a precise control over multiple heterogeneous cell types and material components, which is required to recapitulate physiological functions, and re-establish the human body's fundamental needs. Novel concepts of bioreactor design and material were introduced to enhance the ability to trigger biological signals promoting tissue morphogenesis. Micro-tissues inducing the self-assembly of cells in clusters or organoids is today a reality. In this process, a critical role is played by the ma- 
terial properties (e.g., physico-chemical, structural, mechanical, permeability properties) that can modulate the morphology and functionality of cells, guiding them towards complex 3D tissue architecture.

This Special Issue aims to provide an overview of biomaterials, cells, advanced systems, and devices applied to bioartificial organs in regenerative and reparative medicine. The targeted goal was to highlight how many interesting and valuable opportunities, each highly reliable and efficient in the quest for innovative testing tools and specific therapeutic approaches, are available to tissue engineers today. All the pillars in terms of biomaterials, scaffolds, 3D cell culture, and physiologically relevant bioreactors are presented and discussed. This issue begins with two works reporting innovative approaches to repairing and replacing cells, tissues, and organs that are currently available worldwide to meet the need for patients' organs. The first, in the field of liver tissue engineering, considers induced pluripotent stem cells (iPSCs), which are recognized as one of the most promising candidate therapies. Yao et al. [2021] report a very integrative study showing their potential use in the modeling and treatment of liver disease. The current state of efforts to derive human iPSCs to be used in the bioartificial liver is first presented. Then, strategies based on organ decellularization and aimed to promote cell removal, the preservation of extracellular matrix components, and vascular tree integrity are discussed. They are all particularly challenging to building an implantable vascularized solid organ. The second is based on the evidence that recellularization of a decellularized cadaveric organ scaffold with human cells is currently the most promising approach to building a complex solid vascularized organ. An efficient approach to promoting human-sized wholeliver scaffold decellularization is proposed by Alaby Pinheiro Faccioli et al. [2021]. The success of whole-organ engineering will require the optimization of protocols to produce biologically active scaffolds, the production of the massive quantities of cells needed for recellularization, and the engineering of a bioreactor with physiological stimuli to recapitulate organ function. The production of biologically active scaffolds depends strongly on the optimization of a decellularization protocol.

In the field of heart tissue engineering, current approaches and advances are highlighted by Morrissey et al. [2021]. Novel technological approaches in cardiac tissue repair are represented by $3 \mathrm{D}$ bioprinting that allows for the design of precisely controlled and spatially organized structures, which lead to solutions in tissue repair. This technology is particularly attractive in the design and fab- rication of custom-made devices intended for the maturation of the cardiac tissue, a process that has been shown to increase the viability of implants. Recent progress in $3 \mathrm{D}$ printing of cardiac tissue and valves are summarized in the review by Sedlakova et al. [2021].

Pien et al. [2021] present a critical review on the relationships between fabrication processes and tubular bioartificial organs. The review focuses on hollow tubular organs for the transport of anabolites and catabolites, that is, vessels, trachea, and esophagus, ureter and urethra, and intestine. The design and development of tubular organs is described, taking into account the anatomic hierarchy and their principal physiological functions. 3D bioprinting, solution electrospinning, and melt electrowriting are presented together with their capacity to match the requirements imposed by designing scaffolds compatible with the anatomical and physiologically relevant environment.

Membrane technology offers interesting opportunities for the design of bio/artificial organs and tissue analogues, as well as for drug and cell delivery providing new specific cell therapy with high reliability and efficacy. Nano and micro-structured membranes combined with functional cells can be used for the creation of tissue analogous because of the high control at molecular level of the cell microenvironment. These systems compartmentalize cells in micro- and nano-structured complexes, providing a wide surface area for cell adhesion and ensuring a continuous and selective transport of nutrients and metabolites to and from cells. Interestingly, membranes in fiber configuration with precisely controlled and tunable properties can direct and control the function of different kinds of cells toward the recovery from disorders and injuries. The review by Morelli et al. [2021] summarizes the development of hollow fibers and electrospun fiber membranes used in bioartificial organs, tissue engineered constructs, and in vitro bioreactors, with a special focus on bioartificial liver and neuronal tissue applications.

Polymeric materials such as membranes gain more attention as potential candidates for the repair and development of functional human tissues, such as bone, pancreas, retina, kidney, blood vessels, and nerves. Polycaprolactone/beta-tricalcium phosphate $(\mathrm{PCL} / \beta-\mathrm{TCP})$ obtained by a rotary jet-spinning process are particularly interesting for the repair and regeneration of critical bone, as reported by de Andrade Pinto et al. [2021]. Novel polymeric nanostructures (such as nanofibers, nanorods, nanotubes, etc.), especially block copolymers with distinctive properties [Malik et al., 2021], are among the best studied systems to fit numerous tissue engineering applications 
due to the broad variety of properties they display upon varying the composition ratio and molecular weight of blocks.

Wound healing and cornea regeneration are currently being investigated for improvement of patient outcomes [Poudel et al., 2021]. Biomaterial approaches based on extracellular matrix components like collagen have shown the ability to repair corneal wounds. Other cell-free therapies such as treatment with tolerogenic exosomes and extracellular vesicles may improve treatment outcomes in the case of inflammatory conditions. Many of the technologies described also in combination with cell- or drugbased therapies are promising in the treatment of corneal injuries and disease with a single regenerative solution.

The modeling of organs such as a 3D human placenta is quite challenging. The review by Tutar and Çelebi-Saltik [2021] describes the systems and approaches used in the creation of $3 \mathrm{D}$ placenta, placental transfer models as $3 \mathrm{D}$ placental barriers, and micro-engineered 3D placenta on-a-chip to explore complicated placental responses to nanoparticle exposure.

Altogether, the work collected in this Special Issue emphasize and highlight the latest advances and challenges in the design of bioartificial organs and tissue analogues looking to the future perspectives of tissue engineering and regenerative medicine. We sincerely hope that readers will enjoy reading as much as we enjoyed editing this special issue. The field of bioartificial organs for regen- erative and reparative medicine is definitely advancing, innovation is around the corner, but the most effective strategies have yet to emerge. A focused approach between all partners involved in the development of the field is required. This approach needs to bridge together clinical needs and regulatory requirements, while aligning them with industrial concerns and scientific research outcomes for preserving human life and improving quality of life.

\section{Acknowledgements}

The Editors are grateful to all the authors for their outstanding contribution to the Special Issue.

\section{Conflict of Interest Statement}

The authors have no conflicts of interest to declare.

\section{Funding Sources}

There are no sources of funding to declare.

\section{Author Contributions}

The authors contributed equally to this editorial.

\section{References}

Alaby Pinheiro Faccioli L, Suhett Dias G, Hoff V, Lemos Dias M, Ferreira Pimentel C, Hochman-Mendez, et al. Optimizing the decellularized porcine liver scaffold protocol. Cells TissuesOrgans.2021.doi: 10.1159/000510297. de Andrade Pinto SA, de Nadai Dias FJ, Brasil Camargo Cardoso G, dos Santos Junior AR, de Aro AA, Pino DS, et al. Polycaprolactone/beta-tricalcium phosphate scaffolds obtained via rotary jet-spinning: in vitro and in vivo evaluation. Cells Tissues Organs. 2021. doi: 10.1159/000511570.

Malik S, Sundarrajan S, Hussain T, Nazir A, Ramakrishna $\mathrm{S}$. Role of block copolymers in tissue engineering applications. Cells Tissues Organs. 2021. doi: 10.1159/000511866.
Morelli S, Piscioneri A, Salerno S, De Bartolo L. Hollow fiber and nanofiber membranes in bioartificial liver and neuronal tissue engineering. Cells Tissues Organs. 2021. doi: 10.1159/000511680.

Morrissey J, Mesquita FCP, Hochman-Mendez C, Taylor DA. Whole heart engineering: advances and challenges. Cells Tissues Organs. 2021. doi: 10.1159/000511382.

Pien N, Palladino S, Copes F, Candiani G, Dubruel P, Van Vlierberghe S, et al. Tubular bioartificial organs: from physiological requirements to fabrication processes and resulting properties. A critical review. Cells Tissues Organs. 2021. doi: 10.1159/000519207.
Poudel BK, Robert MC, Simpson FC, Malhotra K, Jacques L, LaBarre P, Griffith M. In situ tissue regeneration in the cornea from bench-tobedside. Cells Tissues Organs. 2021. doi: 10.1159/000514690.https://prod.karger.com/ Article/FulltextPreview/514690.

Sedlakova V, McTiernan C, Cortes D, Suuronen EJ, Alarcon EI. 3D bioprinted cardiac tissues and devices for tissue maturation. Cells Tissues Organs. 2021. doi: 10.1159/000512792.

Tutar R, Celebi-Saltik B. Modeling of artificial 3D human placenta. Cells Tissues Organs. 2021. doi: 10.1159/000511571.

Yao J, Yu Y, Nyberg SL. Induced pluripotent stem cells for the treatment of liver diseases: novel concepts. Cells Tissues Organs. 2021. doi: $10.1159 / 000508182$. 